\title{
Fukuda and Babinski-Weil tests: Within-subject variability and test- retest reliability in nondisabled adults
}

\author{
Nicole Paquet, PhD; ${ }^{1-2 *}$ Anne Taillon-Hobson, $\mathbf{M S c} ;^{1}$ Yves Lajoie, $\mathbf{P h D}^{\mathbf{2}}$ \\ ${ }^{1}$ School of Rehabilitation Sciences and ${ }^{2}$ School of Human Kinetics, Faculty of Health Sciences, University of Ottawa, \\ Ottawa, Canada
}

\begin{abstract}
The Fukuda Stepping Test and the Babinski-Weil test are clinical assessments that presumably reveal unilateral vestibular hypofunction. However, abnormal performances on both of these two tests have been found in nondisabled individuals. This study compared measures of lateral and longitudinal displacements and body rotation for both tests, as well as the within-subject variability and test-retest reliability of the measures. In addition, correlations between hand and foot dominance and these measures were studied. Fifty young, nondisabled participants performed three trials of the 100-step Fukuda test and three trials of the Babinski-Weil test. The testing session was repeated $7 \mathrm{~d}$ later (retest). Lateral displacement, body rotation, and within-subject variability of these two measures were larger on the Fukuda than the Babinski-Weil test. No difference in test-retest reliability was found between the two tests, and a significant correlation was found between body rotation on the Fukuda test and score on the Waterloo Footedness Questionnaire. There may have been smaller variability in results of the Babinski-Weil test because it contains fewer steps (36) than the 100-step Fukuda test. Future research should compare tests with an equal number of steps; in the meantime, the Babinski-Weil test seems to have better psychometric properties than the Fukuda test, at least in nondisabled individuals.
\end{abstract}

Key words: Babinski-Weil test, body rotation, Fukuda Stepping Test, hand and foot dominance, "Marche en étoile" test, test-retest reliability, vestibular hypofunction, vestibulospinal system, Waterloo Footedness Questionnaire, within-subject variability.

\section{INTRODUCTION}

The clinical test battery to assess dynamic balance in patients with vestibular disorders includes the Fukuda Stepping Test and the Babinski-Weil test. The original Fukuda test consists of stepping on the spot 50 or 100 steps while blindfolded with both arms stretched straight forward [1]. The first version of the Babinski-Weil test requires that patients walk forward three steps with eyes closed and then backward three steps, repeated three times [2]. In most patients with unilateral vestibular hypofunction, final position is rotated relative to the initial position on the Fukuda [1,3-6] and Babinski-Weil tests [7]. These two tests are presumed to reveal unilateral vestibular hypofunction via unequal tonic labyrinthine influence upon ipsilateral skeletal muscles. In this case, loading on the right and left lower limbs would be unequal, with more loading on the side of hypofunction, as revealed by a shifted center of pressure toward the side of the vestibular lesion [8]. Asymmetrical lower-limb loading would lead to progressive body turning while stepping on the spot or walking without vision [9].

\footnotetext{
Abbreviations: ICC = intraclass correlation coefficient, Q1 = first quartile, Q3 = third quartile, SD = standard deviation.

*Address all correspondence to Nicole Paquet, PhD; School of Rehabilitation Sciences, University of Ottawa, 451 Smyth Rd, Ottawa, Ontario, H3W 1W5, Canada; 613-5625800, ext 8022; fax: 613-562-5428.
}

Email: npaquet@uottawa.ca

http://dx.doi.org/10.1682/JRRD.2013.09.0206 
The diagnostic value of these tests to detect unilateral vestibular disorders is questionable because both patients with unilateral vestibular disorders and nondisabled adults demonstrate rotation while performing these tests [2,4-5,10-14]. For example, Hickey and colleagues found that 34 percent of 49 nondisabled adults turned leftward and 28 percent turned rightward on the Fukuda test [4]. On average, nondisabled adults turn $45^{\circ}$ on the Fukuda test [11] and $21^{\circ}$ on the Babinski-Weil test [2]. There is a need to further investigate test results in nondisabled individuals, especially on the Babinski-Weil test, for which very few data have been reported.

Clinicians compare a patient's performance results over time and use this as an index of change, notably of improvement. Therefore, the psychometric strength of within-subject variability and test-retest reliability is crucial when multiple performance results are being compared over time. In nondisabled adults, large variability in rotation was found when four trials of the Fukuda test were performed consecutively [9]. The average correlation for two consecutive trials of the Fukuda test was 0.62 for rotation [11], which indicates a moderate correlation [15]. Regarding test-retest reliability, comparison of rotations on the 100-step Fukuda using a 24 h testretest protocol revealed a moderate intraclass correlation coefficient (ICC) of 0.52 [14]. Thus, psychometric qualities of the Fukuda test are medium at best. Our question is whether the Babinski-Weil test's within-subject variability and test-retest reliability, which have not been investigated thus far, are higher than those for the Fukuda test.

It has been suggested that direction of rotation on these two tests could be determined by the side of hand or leg dominance [16]. A significant but small correlation was found between footedness score and rotation on the Fukuda test ( $r=0.26)$ [11]. However, no effect of handedness on rotation direction has been found for the Fukuda test [2,11-12] or the Babinski-Weil test [2]. We propose to further investigate and compare the possible influence of hand and leg dominance on these tests when performed by nondisabled adults.

The first aim of this study was to describe and compare the lateral and longitudinal displacements and body rotation obtained on the Fukuda and Babinski-Weil tests in young nondisabled adults and the within-subject variability of these measures. The second aim was to establish and compare test-retest reliability of these measures for both tests. The third aim was to test for correlations between hand and foot dominance and direction of lateral displacement and rotation for both tests.

\section{METHODS}

\section{Participants}

Fifty nondisabled adults participated in this study. They were 21 males and 29 females between 18 and $36 \mathrm{yr}$ of age (mean \pm standard deviation [SD] $=25 \pm 4 \mathrm{yr}$ ) recruited from among undergraduate and graduate students at the University of Ottawa. Their height was between 1.55 and $1.92 \mathrm{~m}$ (mean $\pm \mathrm{SD}=1.72 \pm 0.09 \mathrm{~m}$ ), and their weight was between 43.0 and $94.5 \mathrm{~kg}$ (mean \pm $\mathrm{SD}=70.0 \pm 13.7 \mathrm{~kg}$ ). They had no previous history of dizziness, vestibulospinal disorder, or balance impairment or recent history of musculoskeletal injury to the lower limbs.

\section{Experimental Procedures}

Experimental sessions occurred in a laboratory setting in which auditory noise was avoided. Two testing sessions were performed separated by $7 \mathrm{~d}$. During the first testing session, the Edinburgh Handedness Inventory [17] and Waterloo Footedness Questionnaire [18] were administered. At test and retest, the Fukuda and Babinski-Weil tests were performed.

For the Fukuda Stepping Test, participants were positioned at the start line and then, after donning custommade opaque ski goggles that occluded vision completely, stepped on the spot for 100 steps. They were in stocking feet and their arms rested comfortably at their sides. The Fukuda testing position of arms positioned in $90^{\circ}$ of shoulder flexion was not used in order to avoid both upper-limb fatigue and any possible variation in arm position within and between participants. The instructions to participants were to stay at the start line while stepping at a comfortable pace.

For the Babinski-Weil test, participants wore the same opaque ski goggles and walked six steps forward (three gait cycles) and then six steps backward, repeating this pattern three times. Thus, a total of 36 steps occurred in a continuous manner. Instructions were to walk on an imaginary straight line and to end the trial as close as possible to the start line. As for the Fukuda test, they were in stocking feet. To prevent the participants from developing a sense of their final position and then attempting to correct their performance in subsequent trials, they were 
guided back to the starting point by an assistant through a complex pathway while still wearing the opaque goggles.

The first 31 participants performed three trials of the Fukuda test and then three trials of the Babinski-Weil test. This performance sequence was reversed for the remaining 19 participants.

\section{Data Collection and Analyses}

Hand dominance was determined with the Edinburgh Handedness Inventory, which gives scores between -100 and 100 [17]. Scores between 51 and 100 indicate righthandedness and scores between -51 and -100 , lefthandedness. Mixed handedness is indicated by scores between -50 and 50. Leg dominance was established with the Waterloo Footedness Questionnaire [18], which gives scores between -20 and 20. Scores between 11 and 20 indicate right-leg dominance and scores between -11 and -20 indicate left-leg dominance. Mixed leg dominance is indicated by scores between -10 and 10 .

For the Fukuda and Babinski-Weil tests, final foot position was marked on the floor with a $20 \mathrm{~cm}$-long piece of masking tape placed just in front of the two big toes after participants had put their feet together. Each mark was identified with the trial number. Three measures were taken from the marks, relative to the start line: lateral displacement in the $x$-axis, longitudinal displacement in the $y$-axis, and body rotation. Measures were taken with a ruler and a goniometer. Lateral displacements to the left and longitudinal displacements behind the start line were given a negative sign. Body rotation was the angle between starting line and final position line. Clockwise rotation (to the right) was given a positive sign and counterclockwise (to the left), a negative sign. Analyses were performed on signed values to allow comparison with previous clinical reports and on absolute values to better describe the extent of distance and rotation errors made by participants.

\section{Statistical Analyses}

All statistical analyses were performed with IBM SPSS Statistics 20 (IBM Corporation; Armonk, New York). For each subject, the three values of lateral and longitudinal displacements and body rotation were used for within-subject variability analyses and then were averaged for further analyses. Mild and extreme outliers were removed from the data set. SPSS identifies outliers using interquartile range, which includes 50 percent of the cases, i.e., third quartile (Q3) minus first quartile (Q1). Values 1.5 times the interquartile range below Q1 or above Q3 are defined as outliers. For signed values, 11 of 300 test and 10 of 294 retest values were removed. For absolute values, 9 of 300 test and 9 of 294 retest values were removed.

Proportions of participants were compared with chisquare tests. Dependent variables obtained for the Fukuda and Babinski-Weil tests were compared with paired $t$-tests or Wilcoxon signed-rank tests. For withinsubject variability, the three trials were compared with repeated measures one-way analysis of variance or Friedman tests. In addition, SDs of the three trials were calculated and then averaged. Mean of SD was compared between the Fukuda and Babinski-Weil tests with Wilcoxon signed-rank tests. Correlations among pairs of the three trials for both tests were assessed with Pearson $r$ or Spearman rho statistics. Test-retest reliability of the three dependent variables obtained for the Fukuda and Babinski-Weil tests was assessed with paired $t$-tests or Wilcoxon signed-rank tests and average measures ICCs [19]. Correlations between Edinburgh Handedness Inventory scores or Waterloo Footedness Questionnaire scores and values of lateral displacement and rotation for both tests were determined with Spearman rho statistics. A probability of 0.05 or less was accepted as significant.

\section{RESULTS}

\section{Test Results}

\section{Signed Values}

Table 1 lists proportion of participants according to the direction of their displacements and body rotations. Null displacement was defined as less than $5 \mathrm{~cm}$ between final position and start line and null body rotation as less than $5^{\circ}$ between final position and start line. A minority of participants, i.e., no more than 19 percent, had null lateral or longitudinal displacement or null body rotation on the Fukuda and Babinski-Weil tests. For each variable, the chi-square test revealed that the proportions of participants with negative, null, and positive values were not significantly different between the Fukuda and BabinskiWeil tests.

For the Fukuda test, mean \pm SD signed values were $-15 \pm$ $87 \mathrm{~cm}$ for lateral displacement, $84 \pm 62 \mathrm{~cm}$ for longitudinal displacement, and $-24^{\circ} \pm 71^{\circ}$ for body rotation. For the Babinski-Weil test, mean \pm SD signed values were $2 \pm$ $43 \mathrm{~cm}$ for lateral displacement, $91 \pm 63 \mathrm{~cm}$ for longitudinal displacement, and $-1^{\circ} \pm 21^{\circ}$ for body rotation. 
Table 1.

Number of participants with negative, null, and positive mean lateral and longitudinal displacement and rotation values on Fukuda Stepping and Babinski-Weil tests.

\begin{tabular}{lcll}
\hline \multirow{2}{*}{ Test } & \multicolumn{3}{c}{ Value, $\boldsymbol{n ( \% )}$} \\
\cline { 2 - 4 } & Negative* $^{*}$ & Null $^{\dagger}$ & Positive \\
\hline Fukuda & $27(54)$ & $7(14)$ & $16(32)$ \\
$\quad$ Lateral Displacement & $4(9)$ & $1(2)$ & $41(89)$ \\
$\quad$ Longitudinal Displacement & $29(59)$ & $2(4)$ & $18(37)$ \\
$\quad$ Body Rotation & & & \\
Babinski-Weil & $21(45)$ & $4(8)$ & $22(47)$ \\
$\quad$ Lateral Displacement & $4(8)$ & $2(4)$ & $44(88)$ \\
$\quad$ Longitudinal Displacement & $20(43)$ & $9(19)$ & $18(38)$ \\
$\quad$ Body Rotation &
\end{tabular}

${ }^{*}$ Final position to left of start line for lateral, behind start line for longitudinal, and counterclockwise rotation (leftward) for rotation.

${ }^{\dagger}$ Distance between final position and start line of $0 \pm 5 \mathrm{~cm}$ for lateral and longitudinal and angular rotation between final position and start line of $0^{\circ} \pm 5^{\circ}$ for rotation.

Because the dependent variables' variance was not homogeneous, Wilcoxon signed-rank tests were used for comparisons. Body rotation tended to be larger on the Fukuda than the Babinski-Weil test $(z=-1.88, p=0.06, r=0.28)$.

\section{Absolute Values}

Figure 1 shows mean + SD absolute values obtained for the three dependent variables on both tests. Lateral displacement $(z=-4.28, p<0.001, r=0.61)$ and body rotation $(z=-5.84, p<0.001, r=0.85)$ were significantly larger on the Fukuda than the Babinski-Weil test.

\section{Within-Subject Variability}

The three trials were used to investigate withinsubject variability. Figure 2 illustrates absolute values obtained on trials 1, 2, and 3 for lateral and longitudinal displacements and body rotation on the Fukuda and Babinski-Weil tests. The mean absolute values of longitudinal displacement obtained on trial 1 of Fukuda were significantly smaller than the values obtained on trials 2 and 3 (Friedman test, $\chi^{2}=14.9, p=0.001$ ). Similar results were found with signed values $\left(\chi^{2}=8.2, p=0.02\right)$.

For each participant and each variable, mean and SD values of the three trials were calculated and further analyses were done on the SDs. Table 2 lists the range of SDs for all participants and the mean of those SDs. Values of SDs were not normally distributed; thus, Wilcoxon signed-rank tests were used for comparisons. Mean of SD for signed values was significantly larger on the Fukuda than the Babinski-Weil for lateral displacement $(z=-3.61, p<0.001, r=0.52)$ and for body rotation $(z=$ (a)

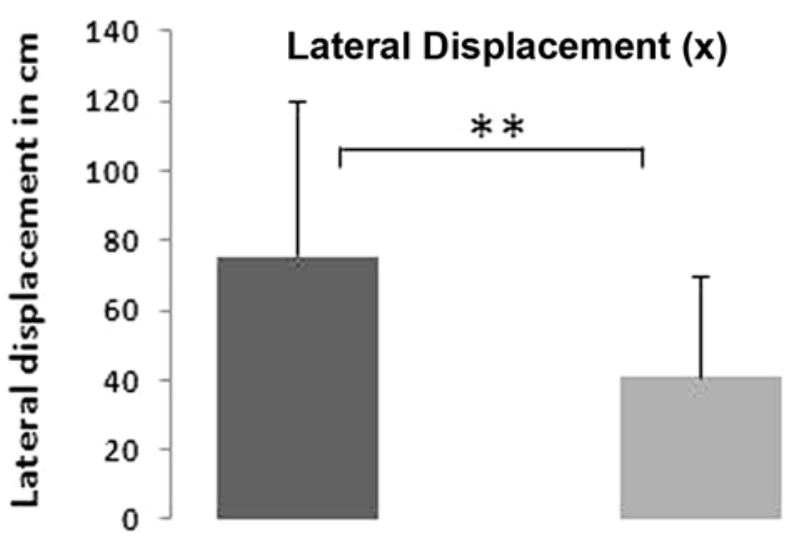

(b)

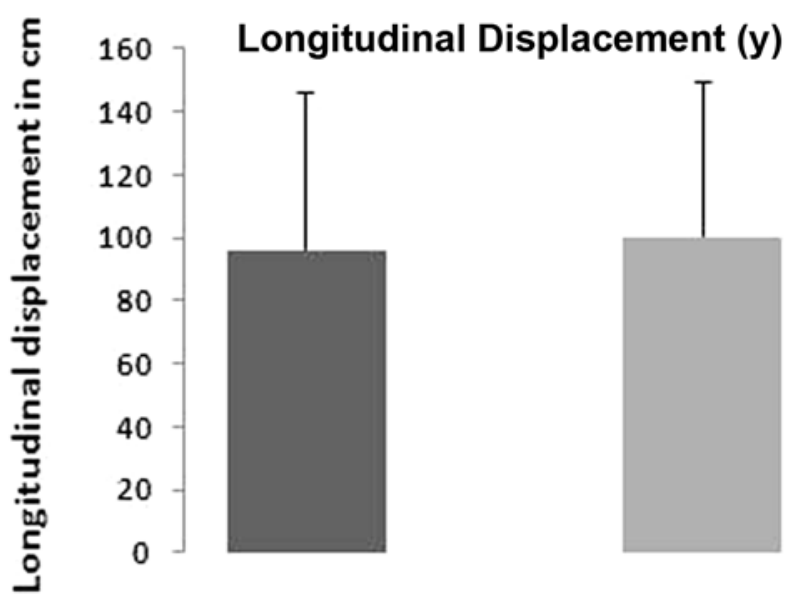

(c)

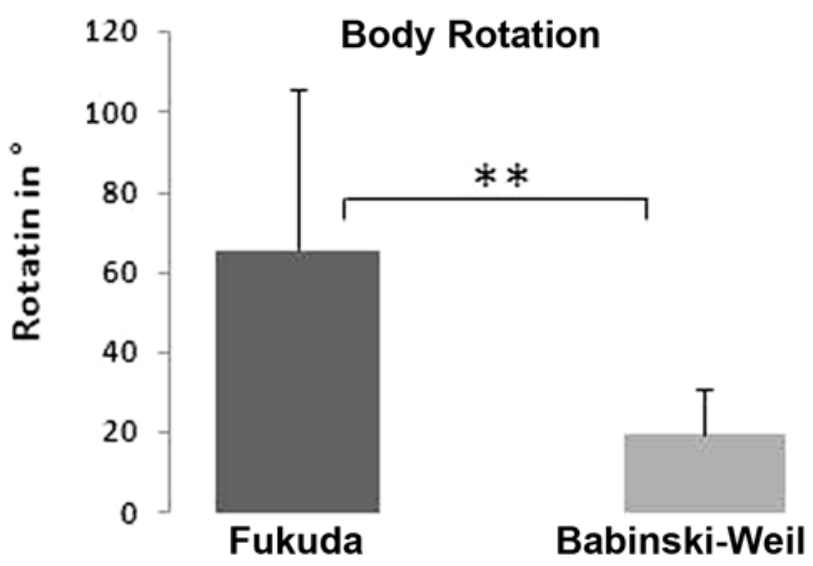

Figure 1.

Absolute values (mean +1 standard deviation) for (a) lateral displacement, (b) longitudinal displacement, and (c) body rotation on Fukuda Stepping and Babinski-Weil tests. ${ }^{* *} p<0.01$.

-5.15, $p<0.001, r=0.74)$. Similar differences were found with absolute values for lateral displacement $(z=-$ 3.56, $p<0.001, r=0.51)$ and for body rotation $(z=-$ 5.29, $p<0.001, r=0.76$ ). 
(a)

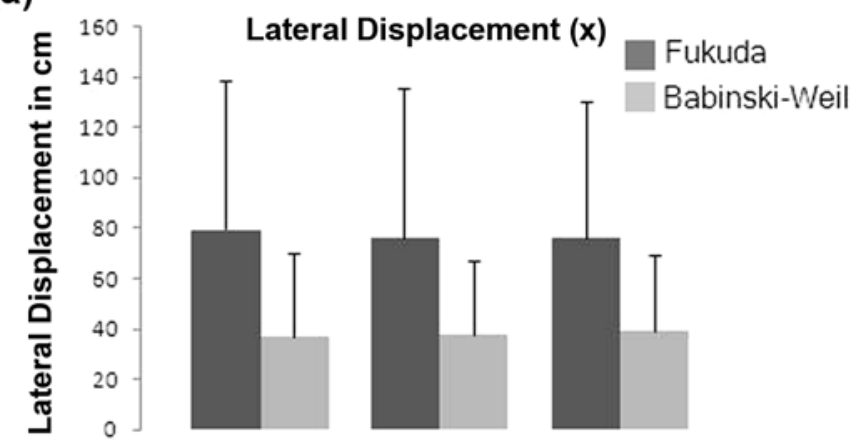

(b)

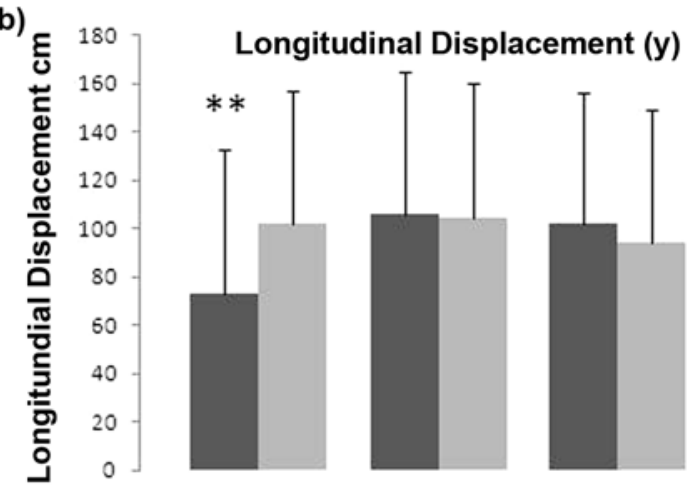

(c)

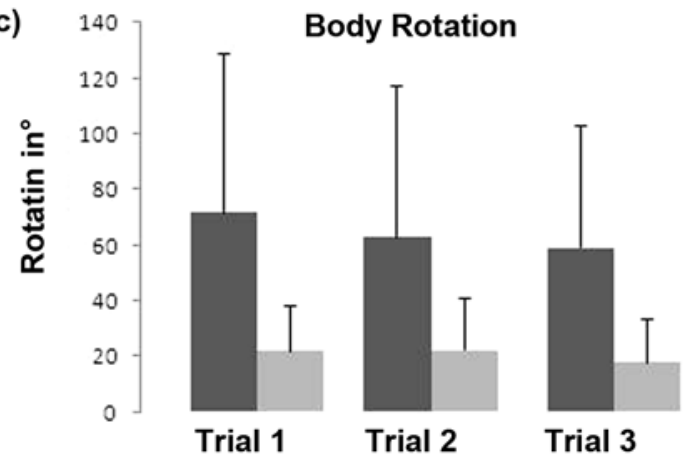

Figure 2.

Absolute values (mean +1 standard deviation) on trials 1,2 , and 3 for (a) lateral displacement, (b) longitudinal displacement, and (c) body rotation on Fukuda Stepping and BabinskiWeil tests. ${ }^{* *} p<0.01$.

Table 3 lists all correlations that were found significant $(p<0.05)$ among pairs of trials. For the Fukuda test, these significant correlations (Pearson $r$ for signed values and Spearman rho for absolute values) ranged from 0.34 to 0.81 , and for the Babinski-Weil test, from 0.39 to 0.80 . Spearman rho was used for absolute values because they were not normally distributed.
Table 2.

Within-subject variability assessed with standard deviation (SD) of three trials of Fukuda Stepping and Babinski-Weil tests.

\begin{tabular}{|c|c|c|c|c|}
\hline \multirow{2}{*}{ Test } & \multicolumn{2}{|c|}{ Range of SD } & \multicolumn{2}{|c|}{ Mean of SD } \\
\hline & Signed & Absolute & Signed & Absolute \\
\hline \multicolumn{5}{|c|}{ Lateral Displacement (cm) } \\
\hline Fukuda & 8-140 & $2-94$ & 45 & 34 \\
\hline Babinski-Weil & $1-83$ & $1-61$ & $24^{*}$ & $19^{*}$ \\
\hline \multicolumn{5}{|c|}{ Longitudinal Displacement (cm) } \\
\hline Fukuda & $4-169$ & $4-145$ & 41 & 37 \\
\hline Babinski-Weil & $4-141$ & $8-58$ & 31 & 28 \\
\hline \multicolumn{5}{|l|}{ Body Rotation $\left({ }^{\circ}\right)$} \\
\hline Fukuda & $2-150$ & 2-112 & 45 & 36 \\
\hline Babinski-Weil & $1-36$ & $1-25$ & $15^{*}$ & $12^{*}$ \\
\hline
\end{tabular}

\section{Test-Retest Reliability}

Table 4 lists mean \pm SD values obtained at test and retest $7 \mathrm{~d}$ later as well as ICCs. For signed values, all paired $t$-tests between dependent variables at test and retest were not significant $(p>0.05)$. For absolute values, lateral displacement at Fukuda retest was significantly smaller than at test $(\mathrm{z}=-2.75, p=0.006, r=0.40)$. ICCs of measures ranged between 0.68 and 0.80 for the Fukuda test and between 0.58 and 0.80 for the BabinskiWeil test.

\section{Correlations Between Dominance and Test Results}

The mean \pm SD Edinburgh Handedness Inventory score was $76.5 \pm 42.9$. Forty-two participants were righthanded (84\%), seven were mixed handed (14\%), and one was left-handed (2\%). The mean \pm SD Waterloo Footedness Questionnaire score was 8.2 \pm 7.3. Twenty participants had right-leg dominance (40\%), twenty-eight had mixed leg dominance (56\%), and two had left-leg dominance (4\%).

Scores on the Edinburgh Handedness Inventory and Waterloo Footedness Questionnaire were not normally distributed, and thus, Spearman rho was calculated to test for correlations. The only significant correlation was between Waterloo Footedness Questionnaire score and signed values of body rotation on the Fukuda test $(r(48)=$ $0.32, p=0.02)$.

\section{Outlier Values}

Table 5 lists outlier values obtained for each measure on the Fukuda and Babinski-Weil tests at test and retest. A total of 39 values out of 1,188 measures were outliers (3.3\% of data set). Sixteen participants had at least one outlier value in their data set. A majority of them, i.e., 11 of 16 , had only one outlier value. This includes when the 
JRRD, Volume 51, Number 6, 2014

Table 3.

Significant correlations among three trials of Fukuda Stepping and Babinski-Weil tests.

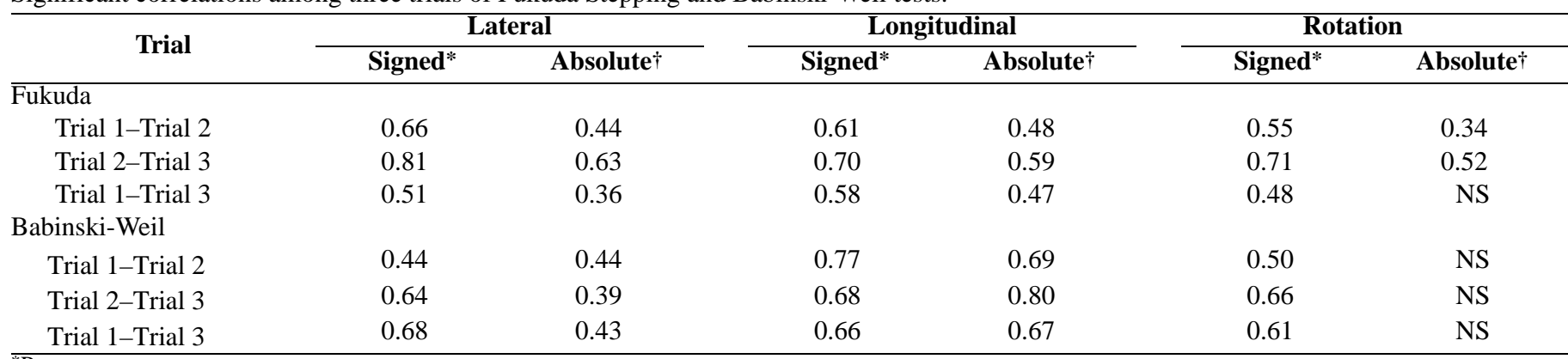

${ }^{*}$ Pearson $r$.

${ }^{\dagger}$ Spearman rho.

NS = not significant

Table 4.

Mean \pm standard deviation (SD) of dependent variables and intraclass correlation coefficients (ICCs) for Fukuda Stepping and Babinski-Weil test results.

\begin{tabular}{|c|c|c|c|c|c|c|}
\hline \multirow{2}{*}{ Test } & \multicolumn{2}{|c|}{ Lateral } & \multicolumn{2}{|c|}{ Longitudinal } & \multicolumn{2}{|c|}{ Rotation } \\
\hline & Signed & Absolute & Signed & Absolute & Signed & Absolute \\
\hline Mean \pm SD Test & $-15 \pm 87$ & $76 \pm 44$ & $84 \pm 62$ & $96 \pm 50$ & $-24 \pm 71$ & $66 \pm 40$ \\
\hline $\mathrm{ICC}^{\dagger}$ & 0.79 & 0.68 & 0.80 & 0.70 & 0.79 & 0.72 \\
\hline $\mathrm{CI}$ & $0.63-0.88$ & $0.43-0.82$ & $0.63-0.89$ & $0.46-0.84$ & $0.62-0.88$ & $0.49-0.85$ \\
\hline \multicolumn{7}{|l|}{ Babinski-Weil } \\
\hline ICC & 0.80 & 0.65 & 0.78 & 0.77 & 0.64 & 0.58 \\
\hline CI & $0.64-0.89$ & $0.36-0.80$ & $0.61-0.88$ & $0.58-0.87$ & $0.34-0.80$ & $0.22-0.77$ \\
\hline
\end{tabular}

${ }^{*} p<0.001$ retest relative to test.

${ }^{\dagger}$ Average measures intraclass correlation.

CI $=95 \%$ confidence interval.

signed and absolute values of the same measure were both identified as outliers. Participant 17 had three outlier values and participant 14, four outlier values. These two participants were women in the group's middle range in terms of age, height, and weight.

\section{DISCUSSION}

This study revealed four main findings. Firstly, measures of lateral displacement and body rotation were larger when participants were performing the Fukuda test than the Babinski-Weil test. Secondly, the within-subject variability of these two measures was larger on the Fukuda than the Babinski-Weil test. Thirdly, test-retest reliability was good and similar for both tests. Lastly, body rotation during the Fukuda test was correlated with leg dominance score on the Waterloo Footedness Questionnaire.

\section{Test Results}

Mean lateral displacement and mean body rotation (absolute values) were smaller during the Babinski-Weil than during the Fukuda test by $35 \mathrm{~cm}$ and $47^{\circ}$, respectively ( $p<0.001)$. This difference in performance on the two tests was expected because the Babinski-Weil test involved only 36 steps, while the Fukuda test consisted of 100 steps. However, mean values of longitudinal displacement (forward displacement) were similar for both tests, i.e., $84 \mathrm{~cm}$ for Fukuda and $90 \mathrm{~cm}$ for BabinskiWeil. A forward displacement of up to $1 \mathrm{~m}$ has previously been reported for the 100-step Fukuda test with the arms stretched straight forward [1]. Arm position does not seem to be a factor in this forward displacement, 
Table 5.

Outlier values obtained at test and retest of Fukuda Stepping and Babinski-Weil tests. Values in parentheses were obtained at retest.

\begin{tabular}{|c|c|c|c|c|c|c|c|c|c|c|c|c|}
\hline \multirow{3}{*}{ Part } & \multicolumn{6}{|c|}{ Fukuda } & \multicolumn{6}{|c|}{ Babinski-Weil } \\
\hline & \multicolumn{2}{|c|}{ Lateral (cm) } & \multicolumn{2}{|c|}{ Longit (cm) } & \multicolumn{2}{|c|}{ Rotation $\left({ }^{\circ}\right)$} & \multicolumn{2}{|c|}{ Lateral (cm) } & \multicolumn{2}{|c|}{ Longit (cm) } & \multicolumn{2}{|c|}{ Rotation ( $\left.{ }^{\circ}\right)$} \\
\hline & Sign & Abs & Sign & Abs & Sign & Abs & Sign & Abs & Sign & Abs & Sign & Abs \\
\hline 2 & & & & & 158 & & & & & & & \\
\hline 4 & & & & & $(-187)$ & & & & & & & \\
\hline 5 & & & & & $(-251)$ & (251) & & & & & & \\
\hline 7 & & 223 & & & & & & & & & & \\
\hline 8 & & & & & & & $(-99)$ & & & & & \\
\hline 11 & & & 337 & 337 & & & & & & & & \\
\hline 13 & & & & & & 176 & & & & & -60 & 60 \\
\hline 14 & (284) & (284) & 362 & 362 & & & $(-137)$ & (137) & & & 65 & 65 \\
\hline 17 & & & & & & & & & & & (136) & (136) \\
\hline 23 & & & & & & & -178 & 178 & & & $97(81)$ & $97(81)$ \\
\hline 29 & & & 288 & $288(301)$ & & & & & & & & \\
\hline 30 & & & & & & & & & & & (79) & (79) \\
\hline 41 & & & -139 & & & & & & & & & \\
\hline 43 & & & & & $(-203)$ & (203) & -108 & & & & & \\
\hline 44 & & & & & & & $(-108)$ & (108) & & & & \\
\hline 47 & & & & & & & -107 & & & & & \\
\hline
\end{tabular}

because similarly large values were found when the arms were on the side [9-10]. For the Babinski-Weil test, a final position in front of the start line was likely due to the use of a shorter step length during backward stepping than forward stepping. Stride length during backward walking at a preferred speed has been reported to be 78 percent of stride length during forward walking [20]. Thus, the three repetitions of forward-backward steps during Babinski-Weil progressively brought participants further and further in front of the start line.

The results for lateral displacement and body rotation performed during the Fukuda test differ from previously reported studies. Our proportion of participants with left displacement (54\%) was larger than the 25 percent found by Zilstorff-Pedersen and Peitersen [10] and left body rotation (59\%) was larger than the 48 percent found by Previc and Saucedo [11]. The mean value of lateral displacement (absolute value of $76 \mathrm{~cm}$ ) was larger than the $25 \mathrm{~cm}$ in observed by Peitersen [9]. Factors that may account for these differences are testing conditions such as starting body position, footwear, and ambient noise. Furthermore, we clearly explained in this article the specific instructions given to participants, which was not always the case in previous studies and may have influenced the results found elsewhere. Finally, our study identified the participants' leg dominance (right, mixed, or left), which was found to significantly correlate with the rotation results for the Fukuda test (see section "Correlations Between Dominance and Test Results" in the “Discussion”).

\section{Within-Subject Variability}

Participants' performance over three trials was quite variable, but more so for the Fukuda than the BabinskiWeil test. Large within-subject variability has been a known characteristic of the Fukuda test [9-11]. Our results add to this body of knowledge; for instance, the SD of body rotation for the three trials of the Fukuda test is $45^{\circ}$ on average (signed values). Significant correlations were found between body rotations on trials 1 and 2, trials 2 and 3, and trials 1 and 3 (Table 3), which confirms previous findings [11]. However, $R^{2}$ ranged from 0.23 to 0.50 , i.e., low- to medium-level correlations [15]. This further indicates that the performance of participants was not consistent from trial to trial. This is a weakness for interpreting the test-retest results of a patient in a clinical setting, especially when only one trial of the Fukuda test is generally performed before and after a clinical intervention.

The Babinski-Weil test was found to have smaller within-subject variability than the Fukuda test for lateral displacement and body rotation (Table 2). It is likely that 
performance variability increases as the number of steps increases, and owing to the fact that the Fukuda test required three times more steps than performed during the Babinski-Weil test, its variability was larger. The results of this study support favoring the Babinski-Weil test over the Fukuda test in the clinical setting because of a better trial-to-trial consistency. However, within-subject variability of the Babinski-Weil test must still be considered quite large, possibly because walking without vision while trying to end up at a specific location requires complex cognitive and sensorimotor integration [21]. Changes in a participant's attention level from trial to trial, for example, could lead to different performances on the test.

\section{Test-Retest Reliability}

Mean values of lateral and longitudinal displacements and body rotation obtained at test and retest performed $7 \mathrm{~d}$ apart were not significantly different for both tests, except for absolute value of lateral displacement on the Fukuda test. ICCs ranged between 0.58 and 0.80 , while a previous study found an ICC of 0.52 for body rotation on the 100-step Fukuda test [14]. Our higher correlation for this measure (ICC $=0.79$ ) was likely due to our larger sample size ( $n=50$ participants) compared with Bonanni and Newton ( $n=30$ participants [14].

Test-retest reliability of blind navigation performance is known to be modest. Distance and direction errors associated with walking without vision toward a previously seen target located $8 \mathrm{~m}$ away were not stable between test and retest executed $7 \mathrm{~d}$ later [22]. Specifically, ICC for body rotation on forward and backward blind navigation were 0.57 and 0.09 , respectively. Because the Babinski-Weil test involved forward and backward walking without vision, there were no expectations that test-retest reliability would be high, i.e., ICCs above 0.80 . Thus, ICCs between 0.64 and 0.80 (signed values, Table 4) indicate that its test-retest reliability is surprisingly good, which confirms an important psychometric quality of this test.

\section{Correlations Between Dominance and Test Results}

The results of this study support those previously reported [11], that there is a significant correlation between body rotation (signed values) on the Fukuda test and score on the Waterloo Footedness Questionnaire, indicating that lower-limb laterality has an influence on the side of rotation during the Fukuda test. However, the effect size of this correlation was small [15], because only 10 percent of body rotation was explained by Waterloo Footedness Questionnaire score. One factor that might explain weakness of this correlation is that 56 percent of participants had a Waterloo Footedness Questionnaire score between -10 and 10 , indicating mixed leg dominance, while only 4 percent of participants had null body rotation on the Fukuda test ( $96 \%$ turned either on the left or on the right, Table 1).

Handedness was not correlated with any Fukuda or Babinski-Weil test results, as has also been reported elsewhere $[2,12,23]$. This suggests that hand dominance is not a factor in turning during stepping or in veering during walking without vision. For instance, no correlation was found between the direction of veering and handedness on blindfolded walking over a distance of up to $150 \mathrm{~m}$ [24]. In addition to this, the large within- and between-subject variability of the Fukuda test that we found could also explain the lack of correlation between these two factors.

\section{Outlier Values}

Performance on the Fukuda and Babinski-Weil tests was poor on infrequent occasions. However, such extreme results do represent a reality in this population and contribute to the questionable validity of these tests. Because similar observations have previously been made when young nondisabled participants were navigating without vision toward a target located $8 \mathrm{~m}$ away [22], it suggests that any locomotion task without vision can be very difficult to execute for some individuals. This is likely because cognition and perception of sensations play important roles in these tasks. Thus, performance can be affected by various factors, such as perception of effort, sensations associated with displacement, or varying level of concentration during the tests.

\section{CONCLUSIONS}

The results of this study indicate that within-subject variability of measures was smaller during the BabinskiWeil than the Fukuda test. Furthermore, test-retest reliability of the Babinski-Weil test was found to be good. These test results and psychometric properties suggest that the Babinski-Weil test is superior to the Fukuda test, at least under the conditions of this study. Future research with nondisabled participants should compare tests in which an equal number of steps is performed. Finally, 
large samples of older adults and patients with vestibular disorders should be investigated to determine which of the two tests provides the most useful results with consistent performance in order to be adopted in the clinical setting.

\section{ACKNOWLEDGMENTS}

\section{Author Contributions:}

Study concept and design: N. Paquet, A. Taillon-Hobson.

Data collection: A. Taillon-Hobson.

Data analysis, statistical analysis, and interpretation of data:

N. Paquet, A. Taillon-Hobson, Y. Lajoie.

Drafting of manuscript: N. Paquet, A. Taillon-Hobson, Y. Lajoie.

Obtained funding: N. Paquet.

Financial Disclosures: The authors have declared that no competing interests exist.

Funding/Support: This material was based on work supported by the University of Ottawa.

Additional Contributions: The authors gratefully acknowledge the assistance of L. Roy for recruitment of participants and data collection. Institutional Review: The University of Ottawa Ethics Board approved this project. All participants signed the informed consent form approved by this Committee.

Participant Follow-up: The authors do not plan to inform participants of the publication of this study.

\section{REFERENCES}

1. Fukuda T. The stepping test: Two phases of the labyrinthine reflex. Acta Otolaryngol. 1959;50(2):95-108.

[PMID:13636842]

http://dx.doi.org/10.3109/00016485909129172

2. Nyabenda A, Briart C, Deggouj N, Gersdorff M. A normative study of the vestibulospinal and rotational tests. Adv Physiother. 2004;6(3):122-29. http://dx.doi.org/10.1080/14038190310012052

3. Moffat DA, Harries ML, Baguley DM, Hardy DG. Unterberger's stepping test in acoustic neuroma. J Laryngol Otol. 1989;103(9):839-41. [PMID:2584872] http://dx.doi.org/10.1017/S0022215100110254

4. Hickey SA, Ford GR, Buckley JG, Fitzgerald O’Connor AF. Unterberger stepping test: A useful indicator of peripheral vestibular dysfunction? J Laryngol Otol. 1990;104(8): 599-602. [PMID:2230549] http://dx.doi.org/10.1017/S0022215100113337

5. Zhang YB, Wang WQ. Reliability of the Fukuda stepping test to determine the side of vestibular dysfunction. J Int Med Res. 2011;39(4):1432-37. [PMID:21986145] http://dx.doi.org/10.1177/147323001103900431
6. Honaker JA, Shepard NT. Performance of Fukuda Stepping Test as a function of the severity of caloric weakness in chronic dizzy patients. J Am Acad Audiol. 2012;23(8): 616-22. [PMID:22967736] http://dx.doi.org/10.3766/jaaa.23.8.6

7. Nyabenda A, Briart C, Deggouj N, Gersdorff M. Benefit of rotational exercises for patients with Meniere's syndrome, method used by the ENT department of St-Luc university clinic. Ann Readapt Med Phys. 2003;46(9):607-14. English, French. [PMID:14642673] http://dx.doi.org/10.1016/j.annrmp.2003.05.001

8. Takemori S, Ida M, Umezu H. Vestibular training after sudden loss of vestibular functions. ORL. 1985;47(2):76-83. [PMID:3872435] http://dx.doi.org/10.1159/000275748

9. Peitersen E. Vestibulospinal reflexes. X. Theoretical and clinical aspects of the stepping test. Arch Otolaryngol. 1967;85(2):192-98. [PMID:6017593] http://dx.doi.org/10.1001/archotol.1967.00760040194011

10. Zilstorff-Pedersen K, Peitersen E. Vestibulospinal reflexes. Spontaneous alterations in the position of normal persons doing the stepping test. Arch Otolaryngol. 1963;77:237-42. [PMID:14003663] http://dx.doi.org/10.1001/archotol.1963.00750010247001

11. Previc FH, Saucedo JC. The relationship between turning behavior and motoric dominance in humans. Percept Mot Skills. 1992;75(3 Pt 1):935-44. [PMID:1454499] http://dx.doi.org/10.2466/pms.1992.75.3.935

12. Reiss M, Reiss G. Asymmetry of the stepping test. Percept Mot Skills. 1997;85(1):305-6. [PMID:9293592] http://dx.doi.org/10.2466/pms.1997.85.1.305

13. Reiss M, Reiss G. Further aspects of the asymmetry of the stepping test. Percept Mot Skills. 1997;85(3 Pt 2):1344-46. [PMID:9450291] http://dx.doi.org/10.2466/pms.1997.85.3f.1344

14. Bonanni M, Newton R. Test-retest reliability of the Fukuda Stepping Test. Physiother Res Int. 1998;3(1):58-68.

[PMID:9718617] http://dx.doi.org/10.1002/pri.122

15. Cohen J. Statistical power analysis for the behavioral sciences. 2nd ed. Hillsdale (NJ): Erlbaum; 1988.

16. Milojevic B, Watson JL. Vestibular asymmetries in rightand left-handed people. Acta Otolaryngol. 1965;60(1-6): 322-30. http://dx.doi.org/10.3109/00016486509127017

17. Oldfield RC. The assessment and analysis of handedness: The Edinburgh inventory. Neuropsychologia. 1971;9(1): 97-113. [PMID:5146491] http://dx.doi.org/10.1016/0028-3932(71)90067-4

18. Elias LJ, Bryden MP, Bulman-Fleming MB. Footedness is a better predictor than is handedness of emotional lateralization. Neuropsychologia. 1998;36(1):37-43. 
JRRD, Volume 51, Number 6, 2014

\section{[PMID:9533385]}

http://dx.doi.org/10.1016/S0028-3932(97)00107-3

19. McGraw KO, Wong SP. Forming inferences about some intraclass correlation coefficients. Psychol Methods. 1996; 1(1):30-46. http://dx.doi.org/10.1037/1082-989X.1.1.30

20. Laufer Y. Age- and gender-related changes in the temporalspatial characteristics of forwards and backwards gaits. Physiother Res Int. 2003;8(3):131-42. [PMID:14533369] http://dx.doi.org/10.1002/pri.281

21. Trullier O, Wiener SI, Berthoz A, Meyer JA. Biologically based artificial navigation systems: Review and prospects. Prog Neurobiol. 1997;51(5):483-544. [PMID:9153072] http://dx.doi.org/10.1016/S0301-0082(96)00060-3

22. Paquet N, Rainville C, Lajoie Y, Tremblay F. Reproducibility of distance and direction errors associated with forward, backward, and sideway walking in the context of blind navigation. Perception. 2007;36(4):525-36.

[PMID:17564199] http://dx.doi.org/10.1068/p5532

23. Toussaint Y, Do MC, Fagard J. What are the factors responsible for the deviation in stepping on the spot? Neurosci
Lett. 2008;435(1):60-64. [PMID:18337006]

http://dx.doi.org/10.1016/j.neulet.2008.02.007

24. Bestaven E, Guillaud E, Cazalets JR. Is “circling” behavior in humans related to postural asymmetry? PLoS ONE. 2012;7(9):e43861. [PMID:22957035] http://dx.doi.org/10.1371/journal.pone.0043861

Submitted for publication September 16, 2013. Accepted in revised form February 7, 2014.

This article and any supplementary material should be cited as follows:

Paquet N, Taillon-Hobson A, Lajoie Y. Fukuda and Babinski-Weil tests: Within-subject variability and testretest reliability in nondisabled adults. J Rehabil Res Dev. 2014;51(6):1013-22.

http://dx.doi.org/10.1682/JRRD.2013.09.0206

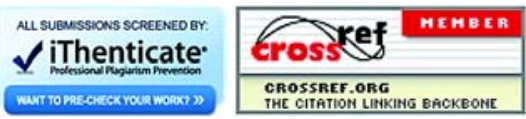

